\title{
Long-term Effect of Continuous Positive Air Pressure Therapy on Intraocular Pressure in Patients with Primary Open-angle Glaucoma with Obstructive Sleep Apnea
}

\author{
Pradtana Hirunpatravong ${ }^{1}$, Tharnsook Kasemsup ${ }^{2}$, Woravipa Na Ayudhya ${ }^{3}$, Polporn Apiwattanasawee ${ }^{4}$
}

\begin{abstract}
Purpose: Prevalence of glaucoma is higher in obstructive sleep apnea (OSA) patients. The gold standard technique to treat OSA is continuous positive airway pressure (CPAP) therapy. The influence of long-term CPAP therapy on intraocular pressure (IOP), blood pressure, ocular perfusion pressure (OPP), and glaucoma progression in primary open-angle glaucoma (POAG) patients was evaluated.

Design: Prospective study.

Materials and methods: In this study, we enrolled 12 eyes from six POAG patients aged $>35$ years, with newly diagnosed OSA and with indication for CPAP therapy. The CPAP was performed for 12 months. We monitored the IOP every 3 months. Visual field was determined at baseline and 12 months.

Results: The mean IOP after CPAP therapy for 12 months was significantly higher than the mean baseline IOP (average IOP 3 months before CPAP therapy) $(19.08 \pm 3.47$ vs $17.83 \pm 2.88 \mathrm{~mm} \mathrm{Hg} ; p=0.006)$. The IOP rising rate was $0.69 \pm 0.47 /$ years $(p=0.138)$ before CPAP therapy and increased to $1.13 \pm 0.47 /$ years $(p=0.016)$ after CPAP therapy. The OPP after 12 months of CPAP was significantly lower than the baseline (42.21 \pm 5.29 vs $45.24 \pm 7.09 \mathrm{~mm} \mathrm{Hg} ; p=0.06$ ). Results showed that the pattern standard deviation (PSD) value of $24-2$ short wavelength automated perimetry (SWAP) visual field was reduced from $5.34 \pm 3.92$ to $4.77 \pm 3.73(p=0.025)$. Antiglaucoma medication was administered to a patient due to increased IOP without glaucoma progression evidence.

Conclusion: The POAG and OSA patients demonstrated significant IOP rising after CPAP therapy but did not show progression of glaucomatous damage. Mean deviation (MD), PSD, and visual field index (VFI) were not significantly different after CPAP therapy.

Précis: Prospective study of POAG and OSA patients demonstrated significant IOP rising after CPAP therapy for a year. The study did not show progression of glaucomatous damage.

Keywords: Continuous positive air pressure, Glaucoma, Intraocular pressure, Obstructive sleep apnea, Primary open-angle glaucoma.

Journal of Current Glaucoma Practice (2019): 10.5005/jp-journals-10078-1262
\end{abstract}

\section{INTRODUCTION}

Obstructive sleep apnea (OSA) is a common sleep-related breathing disorder, which increases the risk of several life-threatening cardiovascular diseases in patients with an apnea-hypopnea index (AHI) score of $\geq 30 .^{1-4}$ Obstructive sleep apnea is characterized by repeated partial or complete upper airway collapse (hypopnea and apnea), gasping episodes, and daytime sleepiness. ${ }^{5}$

Although many studies have demonstrated the potential connection between OSA and glaucoma, their results are considerably inconsistent. ${ }^{6-8}$ A large chart review of 156,336 patients diagnosed with sleep apnea initially showed an increased risk of primary open-angle glaucoma (POAG). However, this difference was not observed in the results of a multivariable analysis that accounted for confounding factors. ${ }^{6}$ However, many studies identified an association between POAG and OSA. Onen et al. ${ }^{7}$ investigated 212 patients with POAG and found that the prevalence of sleep-related breathing disorders (e.g., OSA) was $47.6 \%$. In addition, other studies ${ }^{8-10}$ associated OSA with POAG and ocular hypertension. Patients with glaucoma and OSA exhibited higher intraocular pressure (IOP), worse visual field indices (VFIs), and a thinner retinal nerve fiber layer than the control group. ${ }^{8}$ Following the evaluation of 30 patients with POAG, Mojon et al. ${ }^{9}$ found that the prevalence of OSA was $20 \%$. Moreover, a study involving 16 patients with normal-tension glaucoma (NTG) showed that the prevalence of OSA was $50 \% ;^{10}$ they also found that the

\footnotetext{
${ }^{1,2}$ Department of Ophthalmology, Faculty of Medicine, Vajira Hospital, Navamindradhiraj University, Bangkok, Thailand

${ }^{3,4}$ Department of Otolaryngology, Faculty of Medicine, Vajira Hospital, Navamindradhiraj University, Bangkok, Thailand
}

Corresponding Author: Tharnsook Kasemsup, Department of Ophthalmology, Faculty of Medicine, Vajira Hospital, Navamindradhiraj University, Bangkok, Thailand, Phone: +66 868100403, e-mail: tharnsookk@gmail.com

How to cite this article: Hirunpatravong P, Kasemsup T, Ayudhya WN, et al. Long-term Effect of Continuous Positive Air Pressure Therapy on Intraocular Pressure in Patients with Primary Open-angle Glaucoma with Obstructive Sleep Apnea. J Curr Glaucoma Pract 2019;13(3): 94-98.

Source of support: Faculty of Medicine, Vajira Hospital, Navamindradhiraj University, Bangkok, Thailand

Conflict of interest: None

prevalence of glaucoma among 69 patients with OSA was $7.2 \%$, Notably, this prevalence is significantly higher than that expected in healthy individuals (2\%).

Obstructive sleep apnea is diagnosed using polysomnography. ${ }^{11}$ The severity of the disease is determined using the AHI score/hour, which is the sum of the number of apnea and hypopnea episodes. Apnea is defined as the cessation of the oronasal flow for a duration of $\geq 10$ second. Hypopnea is defined as the reduction in the oronasal 
flow by $>50 \%$ with oxygen desaturation by $3-4 \%$. Most studies have reported that an $\mathrm{AHI}$ score of $\geq 5$ is indicative of OSA. Based on the AHI score, the severity of OSA is classified as follows: mild (AHI score, 5-15), moderate (AHI score, 15-30), and severe (AHI score, $>30$ ).

Sleep apnea can be treated using the standard therapy that comprises the application of continuous positive airway pressure (CPAP) at night to prevent upper airway collapse. ${ }^{12}$ However, many studies have reported that variations in diurnal IOP are high in patients with OSA and that these variations increase after CPAP therapy. Kiekens et al. ${ }^{13}$ measured the 24 -hour IOP (every 2 hours) in 21 patients who were newly diagnosed with OSA and after 1 month of CPAP therapy. They found that CPAP therapy caused an additional increase in IOP, particularly at night. At the baseline, a day before the start of CPAP therapy, fluctuations in the 24-hour IOP (i.e., the difference between the lowest and the highest IOPs) of $\geq 8 \mathrm{~mm} \mathrm{Hg}$ were noted in seven patients. Following 1 month of CPAP therapy, this number increased to 12 patients. The mean difference \pm standard deviation (SD) between the lowest and the highest IOPs at the baseline and those after CPAP therapy was $6.7 \pm$ 1.5 and $9.0 \pm 2.0 \mathrm{~mm} \mathrm{Hg}$, respectively. Another study conducted by Cohen et al. ${ }^{14}$ showed an increase in mean IOP in both the patients who underwent and did not undergo CPAP therapy. Moreover, the mean IOP measured in the sitting position prior to sleeping was $13.33 \pm 2.04$ and $14.02 \pm 2.44 \mathrm{~mm} \mathrm{Hg}$, respectively $(p=0.9)$. After 7 hours of sleep in the supine position, the IOP significantly increased and the mean IOP in patients who underwent and did not undergo CPAP therapy was $19.2 \pm 5.68$ and $19.69 \pm 5.61 \mathrm{~mm} \mathrm{Hg}$, respectively. Notably, after 7 hours of sleep, three patients with OSA and glaucoma treated with CPAP exhibited a mean IOP of $23.75 \mathrm{~mm} \mathrm{Hg}$.

Since the IOP is influenced by OSA and CPAP therapy, previous studies about the effect of treating OSA using CPAP are varied, with most done in a short-term. In the present study, we analyzed IOP and the progression of glaucoma for 1 year after the initiation of CPAP therapy in patients with POAG who were newly diagnosed with OSA to determine the long-term effect of CPAP therapy. Notably, vascular perfusion plays an important role in glaucomatous neuropathy and is frequently reported in OSA. ${ }^{15}$ Therefore, blood pressure and pulse rates were also assessed.

\section{Materials and Methods}

\section{Patients}

This prospective study included 16 eyes from eight patients aged $\geq 35$ years, who were newly diagnosed with OSA based on full-night diagnostic polysomnography. All patients with an AHI score of $\geq 15$ were referred for CPAP therapy.

All enrolled patients had POAG with a controlled IOP for $\geq 3$ months and a normal anterior chamber with an open angle. Patients with any neurological abnormalities, any abnormalities that prevented reliable assessment using applanation tonometry, and previous intraocular surgery, except simple uncomplicated phacoemulsification with an intraocular lens for $>3$ months, were excluded.

All patients provided informed consent prior to their participation in the study. This study was approved by the institutional ethics committee of the Faculty of Medicine, Vajira Hospital, Navamindradhiraj University, Bangkok, Thailand, and was performed according to the tenets of the declaration of Helsinki.

\section{Study Design}

Ophthalmic evaluation was performed for all patients at the baseline. This examination comprised the assessment of medical history, best-corrected visual acuity, and central corneal thickness and slit-lamp biomicroscopy, fundoscopy, gonioscopy, and visual field analysis (Humphrey Field Analyzer; Sita Standard 24-2; Carl Zeiss Meditec, Inc., Dublin, CA). The IOP was measured using a Goldmann applanation tonometer after instilling an anesthetic eye drop ( $0.5 \%$ tetracaine hydrochloride, Alcon). The baseline IOP was defined as the average IOP of 3 months prior to the initiation of CPAP therapy. Moreover, systolic blood pressure (SBP), diastolic blood pressure (DBP), and pulse rate were measured.

The CPAP titration was performed during full-night polysomnography to determine the optimal CPAP pressure, and the CPAP treatment was continued for 1 year.

The ocular perfusion pressure (OPP) is defined as the pressure that forces blood through the ocular vascular bed. The OPP is the difference between the mean arterial and venous pressure. The venous pressure in the eye is almost equivalent to IOP. The OPP was calculated based on IOP, SBP, and DBP, according to the results of ophthalmodynamometric studies as follows: ${ }^{16}$

$$
\mathrm{OPP}=2 / 3[\mathrm{DBP}+1 / 3(\mathrm{SBP}-\mathrm{DBP})]-\mathrm{IOP}
$$

The IOP, SBP, DBP, and pulse rate were recorded every 3 months. Visual field analysis was performed at 12 months after initiating the CPAP therapy.

The primary outcome was a change in IOP 12 months after initiating the CPAP therapy. The secondary outcome was OPP and the progression of glaucoma (worsened visual field and prescription of antiglaucoma drugs).

\section{Statistical Methods}

Data were analyzed using the STATA software (ver. 14.1; StataCorp, College Station, TX, USA). The rates of change in IOP prior to and after CPAP therapy were calculated using multilevel linear regression. Paired $t$ test was performed to compare the differences in IOP, OPP, SBP, DBP, pulse rate, VFI, MD, and pattern standard deviation (PSD) prior to and after CPAP therapy. Mean values were compared using $95 \%$ confidence intervals. A $p$ value of $<0.05$ was considered to indicate statistical significance.

\section{Results}

Sixteen eyes of eight patients with POAG who were newly diagnosed with OSA, in whom CPAP therapy was indicated and continued for 12 months, were included in our study. Of these, four eyes (25\%) were excluded because of intolerance to CPAP therapy (two eyes) and loss to follow-up (two eyes). Finally, 12 eyes from six patients who continued CPAP therapy for 12 months with a compliance of 5.7 hour/night (range: 5-7.3 hour/night) were included in the study.

\section{Characteristics}

The characteristics of patients are shown in Table 1. The mean age \pm SD was $60.6 \pm 4.53$ (range: $52-72$ ) years. Four patients were male (75\%), and the remaining two patients were female (25\%). Two eyes had undergone phacoemulsification with an intraocular lens implantation (16.7\%). Four patients (66.7\%) had hypertension, two patients (33.3\%) had dyslipidemia, two patients (33.3\%) had atrial fibrillation, and one patient (16.7\%) had diabetes without diabetic 
retinopathy. The average $\mathrm{AHI}$ score was $37.42 \pm 15.62$ per hour prior to CPAP treatment. This value reduced to $2.53 \pm 1.55$ /hour after CPAP treatment.

\section{Intraocular Pressure}

Twelve months after the initiation of CPAP therapy, the mean IOP significantly increased compared with that at the baseline (19.08 \pm 3.47 vs $17.83 \pm 2.89 \mathrm{~mm} \mathrm{Hg}$, respectively; $p=0.006$ ). In addition, the increase in mean IOP at 3,6 , and 9 months was $17.17 \pm 2.63$ $(p=0.05), 18.58 \pm 3.68(p=0.27)$, and $17.50 \pm 3.40(p=0.56)$, respectively (Table 2 ).

Baseline IOP rising rate was $0.69 \pm 0.47 \mathrm{~mm} \mathrm{Hg} /$ year $[95 \%$ confidence interval $(\mathrm{Cl}):-0.18$ to $0.13 ; p=0.138]$, whereas it was $1.13 \pm 0.47 \mathrm{~mm} \mathrm{Hg} /$ year after CPAP therapy (95\% Cl: $0.21-2.05$; $p=0.016)$. The difference in IOP after CPAP therapy $(1.13 \pm 0.47$ $\mathrm{mm} \mathrm{Hg}$ /year) was more than the baseline $(0.69 \pm 0.47 \mathrm{~mm} \mathrm{Hg} /$ year $)$ but was not statistically significant $(p=0.059)$ (Fig. 1).

Table 1: Patient characteristics

\begin{tabular}{lcl}
\hline & Mean \pm SD & Range \\
\hline Age & $60.6 \pm 4.53$ & $52-72$ \\
$\mathrm{BMI}\left(\mathrm{kg} / \mathrm{m}^{2}\right)$ & $26.6 \pm 1.84$ & $24.4-31.5$ \\
$\mathrm{AHI}$ ( per hour) & $37.42 \pm 15.62$ & $17.2-67.3$ \\
VA (LogMAR) & $0.11 \pm 0.22$ & $0-0.6$ \\
CCT (microns) & $582.31 \pm 33.94$ & $546-651$ \\
\hline
\end{tabular}

$\mathrm{BMI}$, basal metabolic index; $\mathrm{AHI}$, apnea-hypopnea index; $\mathrm{n} / \mathrm{h}$, number per hour; CCT, central corneal thickness

Table 2: Mean IOP at 3, 6, 9, and 12 months after the initiation of CPAP therapy compared with baseline IOP

\begin{tabular}{llll}
\hline & $I O P(\mathrm{~mm} \mathrm{Hg})$ & $95 \% \mathrm{Cl}$ & $p$ value \\
\hline 3 months & $17.17 \pm 2.63$ & -0.02 to 1.33 & $0.05^{*}$ \\
6 months & $18.58 \pm 3.68$ & -2.16 to 0.66 & 0.27 \\
9 months & $17.50 \pm 3.40$ & -0.88 to 1.55 & 0.56 \\
12 months & $19.08 \pm 3.48$ & -2.06 to $(-0.43)$ & $0.006^{*}$ \\
\hline
\end{tabular}

$\mathrm{IOP}$, intraocular pressure; CPAP, continuous positive airway pressure ${ }^{*} p$ value $\leq 0.05$ which suggest statistically significant

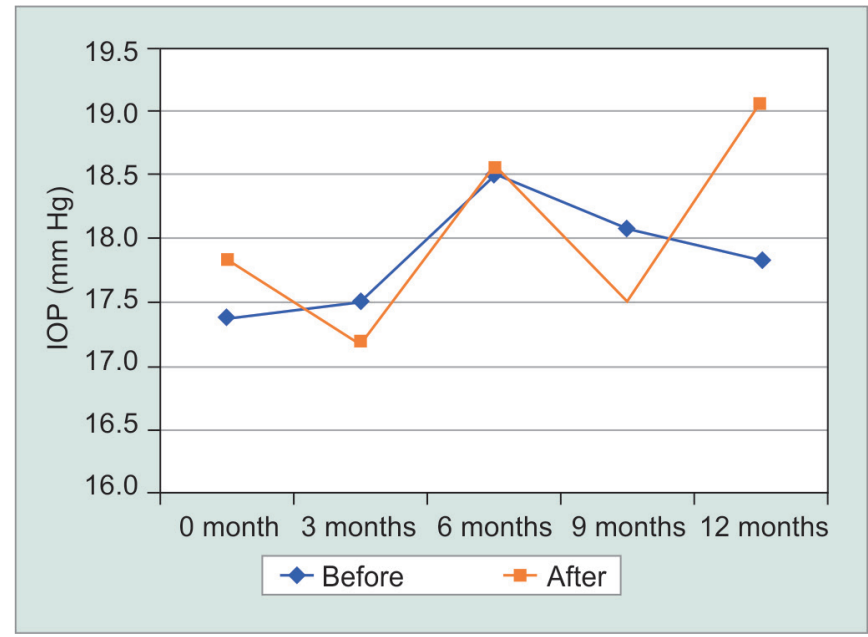

Fig. 1: Comparison of intraocular pressure curves prior to the initiation of continuous positive airway pressure therapy and throughout the 12 months after initiating CPAP therapy (multilevel linear regression)

\section{Progression of Glaucoma}

The results showed that the PSD value of the 24-2 SWAP visual field significantly reduced from $5.33 \pm 3.92$ to $4.77 \pm 3.73(p=0.03)$. The $M D$ was reduced from $-5.29 \pm 5.09$ to $-5.43 \pm 4.97(p=0.81)$. The VFI increased from $86.75 \pm 13.9$ to $87.67 \pm 14.62(p=0.54)$.

\section{Hemodynamics}

The paired $t$ test showed that the difference between mean OPP \pm SD at the baseline and that at 12 months after initiating CPAP therapy was not statistically significant $(45.24 \pm 7.09$ vs $42.21 \pm$ 5.29 , respectively; $p=0.06$ ). Compared with those at the baseline, SBP and DBP decreased at 12 months after initiating CPAP therapy (for SBP; from $123.17 \pm 8.56 \mathrm{~mm} \mathrm{Hg}$ to $122.50 \pm 10.70 \mathrm{~mm} \mathrm{Hg}$; $p=0.81$ and for DBP; from $80.33 \pm 8.69 \mathrm{~mm} \mathrm{Hg}$ to $76.67 \pm 6.02$ $\mathrm{mm} \mathrm{Hg} ; p=0.18$ ) (Table 3 ). Moreover, there was a statistically significant decrease in mean pulse rate \pm SD from $83.00 \pm 13.28$ beats per minute $(\mathrm{bpm})$ to $72.33 \pm 4.42 \mathrm{bpm}(p=0.01)$.

\section{Discussion}

In the present study, we evaluated the long-term effect of the first use of CPAP therapy on IOP in patients with POAG who were newly diagnosed with OSA. Studies investigating the relationship between sleep apnea, glaucoma, and CPAP therapy were mostly conducted during the night and over a short period of time. Very few studies have evaluated the effect of CPAP on the risk of glaucoma in patients with OSA. To the best of our knowledge, this is the first study to investigate changes in IOP, hemodynamic parameters, and the progression of glaucoma following long-term CPAP therapy (1 year). The strengths of the present study include its prospective design, the 12-month follow-up period, and the low rate of discontinuation (25\%).

Numerous studies have reported that variations in diurnal IOP are higher in patients with OSA and increase further after CPAP therapy. Cohen et al. ${ }^{14}$ also reported an increase in mean IOP to $23.75 \mathrm{~mm} \mathrm{Hg}$ after 7 hours of sleep in three patients with obstructive sleep apnea syndrome (OSAS) with glaucoma treated with CPAP therapy. Moreover, Kiekens et al. ${ }^{13}$ observed a significant increase in overnight IOP after 1 month of CPAP therapy from that at the baseline. Regarding fluctuations in 24-hour IOP, the mean difference between the lowest and highest IOP was $6.7 \pm 1.5 \mathrm{~mm}$ $\mathrm{Hg}$ at the baseline that increased to $9.0 \pm 2.0 \mathrm{~mm} \mathrm{Hg}$ after CPAP therapy. The results showed that CPAP therapy did not influence IOP while patients were awake. In our study, the difference between mean IOP at the baseline and that at 12 months after initiating CPAP therapy was statistically significant. The IOP was measured

Table 3: Mean systolic and diastolic blood pressure, pulse rate, and ocular perfusion pressure at baseline and 12 months after the initiation of CPAP therapy

\begin{tabular}{llll}
\hline & Baseline & $\begin{array}{l}\text { 12 months after } \\
\text { CPAP }\end{array}$ & p value \\
\hline $\begin{array}{l}\text { Systolic blood } \\
\text { pressure (mm Hg) }\end{array}$ & $123.17 \pm 8.56$ & $122.50 \pm 10.70$ & 0.81 \\
$\begin{array}{l}\text { Diastolic blood } \\
\text { pressure (mm Hg) }\end{array}$ & $80.33 \pm 8.69$ & $76.67 \pm 6.02$ & 0.18 \\
$\begin{array}{l}\text { Pulse rate (beats } \\
\text { per minute) }\end{array}$ & $83.00 \pm 13.28$ & $72.33 \pm 4.42$ & $0.01^{*}$ \\
$\begin{array}{l}\text { Ocular perfusion } \\
\text { pressure }\end{array}$ & $45.24 \pm 7.09$ & $42.21 \pm 5.29$ & $0.06^{*}$ \\
\hline
\end{tabular}

CPAP, continuous positive airway pressure ${ }^{*} p$ value $\leq 0.05$ suggests statistical significant 
using applanation tonometry during office hours. The results of the present study are consistent with those of previous studies even though diurnal variation or supine position was not used or frequent awakening of the patient to perform IOP measurements was not performed in our study.

The reason for the observed increase in IOP after CPAP therapy is still unclear. It is suggested that CPAP therapy leads to an increase in the intrathoracic pressure, which results in the elevation of pressure in the venous circulation. This effect may reduce the aqueous humor outflow through the episcleral veins, thus explaining the observed increase in IOP.

Contradictory to the results of the present study, Pépin et al. ${ }^{17}$ observed an improvement in 24-hour IOP following the initiation of CPAP therapy. They found that CPAP therapy decreases nighttime and daytime IOP. Both the IOP and blood pressure were measured every hour for 24 hours in 18 patients at the time of OSAS diagnosis and after 1 month of CPAP therapy. The authors concluded that CPAP therapy may exert a beneficial effect on glaucoma in patients with OSAS.

Obstructive sleep apnea causes insufficient blood supply and nourishment to the retinal nerve fiber layer (RNFL) and optic nerve. ${ }^{18}$ The CPAP therapy is known to ameliorate hemodynamic parameters in patients with OSA by decreasing hypoxia and the risk of damage due to vascular perfusion. However, our study revealed a decrease in OPP from $45.24 \pm 7.09$ at the baseline to $42.21 \pm 5.29$ at 12 months after initiating CPAP therapy, with $p$ value of 0.06 . Decreases in OPP may be a potential risk factor for a higher prevalence of glaucoma. In the present study, the patients examined were in a seated position. Liu et al. ${ }^{19}$ found that nocturnal OPP in the supine position was significantly higher than diurnal OPP in the seated position. These results are inconsistent with the OPP findings reported in healthy individuals.

Kiekens et al. ${ }^{13}$ also reported that the increase in nocturnal IOP is enhanced and paralleled by a decrease in OPP during CPAP therapy at night. In contrast to the OPP findings observed in healthy individuals, Liu et al. ${ }^{19}$ showed that nocturnal OPP in the supine position was significantly higher than diurnal OPP in the seated position.

In a retrospective cohort study conducted in Taiwan using data from the Longitudinal Health Insurance Database 2000, Chen et al. stated the treatment options for OSA and identified the risk factors associated with the development of glaucoma. The study included a large sample of patients with OSA $(n=2,528)$ and randomly selected age-matched controls $(n=10,112)$. The results showed that CPAP therapy did not reduce the risk of glaucoma in patients with OSA compared with that in healthy controls (hazard ratio = $1.65,95 \% \mathrm{Cl}: 1.09-2.49) .^{20}$

The current results show that CPAP therapy does not accelerate the progression of glaucoma. Kadyan et al. ${ }^{21}$ found that the prevalence of glaucoma in their study was similar to that observed in healthy individuals $(2 \%, p=0.429)$. They speculated that this observation may be related to the use of CPAP in the majority of patients with OSA.

In a case study involving patients with NTG and OSAS, the progression of glaucoma ceased after 3.5 years of CPAP therapy. This effect was directly attributed to the therapy. ${ }^{22}$

The present results showed that CPAP therapy did not reduce the risk of glaucoma, which was correlated with IOP or OPP, in patients with OSA. However, these data cannot determine whether the use of CPAP therapy exerts a deleterious effect on the development or progression of glaucoma.
The limitations of the present study include the small sample size and absence of a matched control group. Furthermore, visual field damage may be detected 12 months after optic nerve damage from CPAP therapy. Because of the small sample size, it was not possible to evaluate the association between glaucoma and the severity of OSA as a potential risk factor. However, the number of patients included in most of the previous studies was limited.

In conclusion, the results of this study showed that CPAP therapy did not reduce the risk of glaucoma. Patients with OSA demonstrated significant increases in IOPs and decreases in OPPs after CPAP therapy. In this study, MD, PSD, and VFI did not demonstrate significant differences after CPAP therapy. Hence, the current results support the notion that CPAP therapy does not accelerate the progression of glaucoma. However, the present findings cannot determine whether the use of CPAP therapy exerts a deleterious or beneficial effect on the progression of glaucoma. Therefore, patients with OSA receiving CPAP therapy should undergo regular screening for glaucoma and the IOP monitoring.

\section{ACKNOWLedgment}

The authors would like to thank Enago (www.enago.com) for the English-language review.

\section{References}

1. Punjabi NM, Caffo BS, Goodwin JL, et al. Sleep-disordered breathing and mortality: a prospective cohort study. PLoS Med 2009;6(8):e1000132. DOI: 10.1371/journal.pmed.1000132.

2. Shivalkar B, Van de Heyning C, Kerremans M, et al. Obstructive sleep apnea syndrome: more insights on structural and functional cardiac alterations, and the effects of treatment with continuous positive airway pressure. J Am Coll Cardiol 2006;47(7):1433-1439. DOI: 10.1016/j.jacc.2005.11.054.

3. Shahar E, Whitney CW, Redline S, et al. Sleep-disordered breathing and cardiovascular disease: cross-sectional results of the sleep heart health study. Am J Respir Crit Care Med 2001;163(1):19-25. DOI: 10.1164/ajrccm.163.1.2001008.

4. Shamsuzzaman AS, Gersh BJ, Somers VK. Obstructive sleep apnea: implications for cardiac and vascular disease. JAMA 2003;290(14):1906-1914. DOI: 10.1001/jama.290.14.1906.

5. Olson EJ, Moore WR, Morgenthaler TI, et al. Obstructive sleep apneahypopnea syndrome. Mayo Clin Proc 2003;78(12):1545-1552. DOI: 10.4065/78.12.1545.

6. Stein JD, Kim DS, Mundy KM, et al. The association between glaucomatous and other causes of optic neuropathy and sleep apnea. Am J Ophthalmol 2011;152(6):989.e3-998.e3. DOI: 10.1016/ j.ajo.2011.04.030.

7. Onen $\mathrm{SH}$, Mouriaux F, Berramdane L, et al. High prevalence of sleepdisordered breathing in patients with primary open-angle glaucoma. Acta Ophthalmol Scand 2000;78(6):638-641. DOI: 10.1034/j.16000420.2000.078006638.x.

8. Moghimi S, Ahmadraji A, Sotoodeh $\mathrm{H}$, et al. Retinal nerve fiber thickness is reduced in sleep apnea syndrome. Sleep Med 2013;14(1):53-57. DOI: 10.1016/j.sleep.2012.07.004.

9. Mojon DS, Hess CW, Goldblum D, et al. Primary open-angle glaucoma is associated with sleep apnea syndrome. Ophthalmologica 2000;214(2):115-118. DOI: 10.1159/000027478.

10. Mojon DS, Hess CW, Goldblum D, et al. Normal-tension glaucoma is associated with sleep apnea syndrome. Ophthalmologica 2002;216(3):180-184. DOI: 10.1159/000059625.

11. Deegan PC, McNicholas WT. Pathophysiology of obstructive sleep apnea. Eur Respir J 1995;8(7):1161-1178. DOI: 10.1183/ 09031936.95.08071161.

12. Indications and standards for use of nasal continuous positive airway pressure (CPAP) in sleep apnea syndromes. American Thoracic 
Society. Official statement adopted March 1944. Am J Respir Crit Care Med 1994;150(6 Pt 1):1738-1745. DOI: 10.1164/ajrccm.150.6.7952642.

13. Kiekens S, De Groot V, Coeckelbergh T, et al. Continuous positive airway pressure therapy is associated with an increase in intraocular pressure in obstructive sleep apnea. Invest Ophthalmol Vis Sci 2008;49(3):934-940. DOI: 10.1167/iovs.06-1418.

14. Cohen Y, Ben-Mair E, Rosenzweig E, et al. The effect of nocturnal CPAP therapy on the intraocular pressure of patients with sleep apnea syndrome. Graefes Arch Clin Exp Ophthalmol 2015;253(12):2263-2271. DOI: 10.1007/s00417-015-3153-5.

15. Baguet JP, Pépin JL, Hammer L, et al. Cardiovascular consequences of obstructive sleep apnea syndrome. Rev Med Interne 2003;24(8):530537. DOI: 10.1016/S0248-8663(03)00142-5.

16. Sehi M, Flanagan JG, Zeng L, et al. Relative change in diurnal mean ocular perfusion pressure: a risk factor for the diagnosis of primary open-angle glaucoma. Invest Ophthalmol Vis Sci 2005;46(2):561-567. DOI: 10.1167/iovs.04-1033.

17. Pépin JL, Chiquet $C$, Tamisier $R$, et al. Frequent loss of nyctohemeral rhythm of intraocular pressure restored by nCPAP treatment in patients with severe apnea. Arch Ophthalmol 2010;128(10):1257-1263. DOI: 10.1001/archophthalmol.2010.220.

18. Agarwal R, Gupta SK, Agarwal P, et al. Current concepts in the pathophysiology of glaucoma. Indian J Ophthalmol 2009;57(4): 257-266. DOI: 10.4103/0301-4738.53049.

19. Liu JH, Gokhale PA, Loving RT, et al. Laboratory assessment of diurnal and nocturnal ocular perfusion pressures in humans. J Ocul Pharmacol Ther 2003;19(4):291-297. DOI: 10.1089/108076803322279354.

20. Chen HY, Chang YC, Lin CC, et al. Obstructive sleep apnea patients having surgery are less associated with glaucoma. J Ophthalmol 2014;2014:838912. DOI: 10.1155/2014/838912.

21. Kadyan A, Asghar J, Dowson L, et al. Ocular findings in sleep apnoea patients using continuous positive airway pressure. Eye (Lond) 2010;24(5):843-850. DOI: 10.1038/eye.2009.212.

22. Kremmer S, Selbach JM, Ayertey HD, et al. Normal tension glaucoma, sleep apnea syndrome and nasal continuous positive airway pressure therapy-case report with a review of literature. Klin Monbl Augenheilkd 2001;218(4):263-268. DOI: 10.1055/s-200114923. 